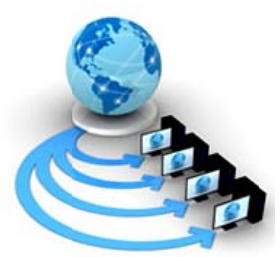

Volume 9, No. 1, January-February 2018

International Journal of Advanced Research in Computer Science

RESEARCH PAPER

\title{
TO ENHANCE THE FEATURE EXTRACTION BABC ALGORITHM TOIMPROVE THE CLASSIFICATION OF MULTIDIMENSIONAL ACCURACY
}

\author{
Dr. K.Mani \\ Associate Professor in Computer Science, \\ Nehru Memorial College, Puthanampatti, Trichy
}

\author{
N. Elavarasan \\ Research Scholar in Computer Science, \\ Nehru Memorial College, Puthanampatti, Trichy
}

\begin{abstract}
Presently a days people are more intrigued to express and offer their perspectives, feedback's, recommendations, and assessments about a specific point on the web. People and company rely more on online opinions about products and services for their decision making criterionreferenced tests, classification consistency and accuracy are viewed as important indicators for evaluating reliability and validity of classification results. Numerous attributes, procedures have been proposed in the framework of the unidimensional item response theory of estimate these indices. Some of these were based on total sum scores, others on latent trait estimates. However, there exist very few attempts to develop them in the framework of multidimensional item response theory. Based on previous studies, the aim of this study is first to estimate the consistency and accuracy indices of multidimensional ability estimates from a single administration of a criterion-referenced test.A noteworthy issue in distinguishing the multidimensional grouping is the high dimensional of the component extraction. The majority of these highlights in insignificant, repetitive, and loud, which influences the execution of the classifier. Along these lines, include extraction is a basic advance in the phony audit location to decrease the dimensional of the component space and to enhance precision. In this paper, double fake honey bee province $(\mathrm{BABC})$ with $\mathrm{KNN}$ is proposed to take care of highlight extraction issues for assumption grouping. The exploratory outcomes exhibit that the proposed strategy chooses more enlightening highlights set contrasted with the aggressive strategies as it accomplishes higher characterization exactness $96.00 \%$.
\end{abstract}

KeyWords: Feature extraction, BABC, K-NN, Classification accuracy.

\section{INTRODUCTION}

Highlight extraction assumes an indispensable part to evacuate loud, unimportant or excess featuresfrom the dataset[1]. This undertaking enhances the exactness and diminishes the computational cost[2]. Numerous transformative algorithms[3] have been utilized for highlight extraction, which incorporates hereditary calculations and swarm calculations. Swarm Insight calculations, for example, properties, Insect State Improvement (ACO), Molecule Swarm Enhancement (PSO)[5],Bat Calculation (BAT), and Manufactured Honey bee Province. The Element extractionprocess can be sorted into two stages, namelythe channel and the wrapper approach[4]. The channel demonstrates dissects the characteristic properties of information free any Learning calculations. The channel strategy can perform both subset extraction and positioning. Positioning includes distinguishing the significance of the considerable number of highlights. In any case, this technique is unequivocally utilized as a pre-process strategy since it chooses repetitive highlights. The wrapper display dissimilar to other channel approach, thinks about the connection between highlights. This technique at first uses an upgrading calculation to create different subsets of highlights and after that uses an order BABC calculation to break down the subsets generated[11]. Because of the highdimensional component space, choosing the ideal element subset is a NP-finish problem[18]. With a specific end goal to tackle extensive scale highlight extraction issues, conventional streamlining calculation isn't effective. In this way, meta-heuristic calculations have been widely connected to illuminate the component extraction problem[19].

In 2005, Karaboga created Simulated Honey bee Settlement
(ABC) calculation which is another populace based metaheuristic swarm savvy BABC calculation. It depends on their rummaging conduct of honey bees. Simulated honey bee province calculation comprises of three sorts of honey bees and they are representative honey bee, spectator honey bee and scout honey bees. Worker honey bee is one that perceives the sustenance sources, relying on the productivity. It conveys all the data about the sustenance source to the hive where the spectator honey bees are pausing. The worker honey bee plays out its moving ceremonies previously the passerby honey bees. Each unique move of the representative honey bee speaks to an alternate bearing of the sustenance source from the hive. With the informationobtained from the worker honey bees the spectator honey bees select the nourishment source with the greatest benefit. Presently the worker honey bee begins to gather nourishment from the source as long as the sustenance source winds up plainly depleted. Once the nourishment source is depleted the representative honey bee overlooks the past data and turns into an investigates honey bee and set in scan for new sustenance sources haphazardly. In this paper, we proposed a parallel simulated honey bee settlement [13-16] based element extraction (BABC) strategy to choose an ideal component subset from the informational collection. The remaining of the paper is organized as follows: Section II describes the related works, Section III describes the methodology of the proposed work, Section IV describes the Experimental results and discussions of the proposed method and finally the Section $\mathrm{V}$ describes the concludes of the proposed work. 


\section{RELATED WORKS}

In addition, a Binary Code-based Artificial Bee Colony (BABC)[7]mechanism was proposed for resolving the issues that emerge during the construction of the multicast spanning tree. This BABC approach uses a two element deviation method that aids in maintaining the consistency of the solutions that are optimally coded using binary values[12]. The proposed BABCscheme is determined to tackle the issues that arise due to the congestive conditions of the network. BABC is found to determine the multicast spanningtree with a success rate of $92 \%$ by generating a number of sub optimal solutions in each iteration[8]. BABC is also adaptable when the tree pathsof the network are necessarily re-built under dynamic network changes. Artificial Bee Colony based Multicast Routing (ABCMR) was proposed for determining multicast tree that aids in effective group communication. The onlooker, employee and scout bee phases of $\mathrm{ABC}$ are suitably applied in multicast routing to determine the routes of the multicast network based on the requirements met in minimizing jitter, latency and cost. The results of ABCMR infer its potential in ensuring optimal solutions in each and every generation, such that optimal levelof discrimination between solutions can be performed effectively[17].A highly efficient and accurate model for modeling nonlinear systems called the NARMAX[9]model was introduced as a unified and accurate representation for a wide variety of nonlinear systems[19].

\section{METHODOLOGY}

It is conceivable to specifically arrange a trait utilizing the multi-dimensional class display. The class of the report for each measurement is resolved autonomously. We called this multidimensional-based characterization. Contrasted BABC and KNN classification[10], the granularity of multidimensional arrangement is coarser. For each measurement, it groups a traits in light of classifications in that measurement as opposed to characterizing it into the arrangement of better classes as done in level order. Despite the fact that the multidimensional classification hasn't accurately spoken to any better classes, the quantity of preparing information Traits per class is generally high per testing information. As an outcome, multi-dimensional characterization increases high precision of each measurement and results in high exactness for the general grouping exactness when there are few preparing information. It additionally performs quicker than BABC based grouping since there are less classes to be looked at KNN calculations. To examine the proficiency of multidimensional grouping of the multidimensional classification demonstrate, $\mathrm{KNN}$ based BABC test and preparing system surely understood arrangement calculations called k-closest neighbors (k-NN), BABC and highlight extraction approaches are applied.In request to remove protest highlights, we require an Order that has experienced Characterization division and any essential morphological sifting.

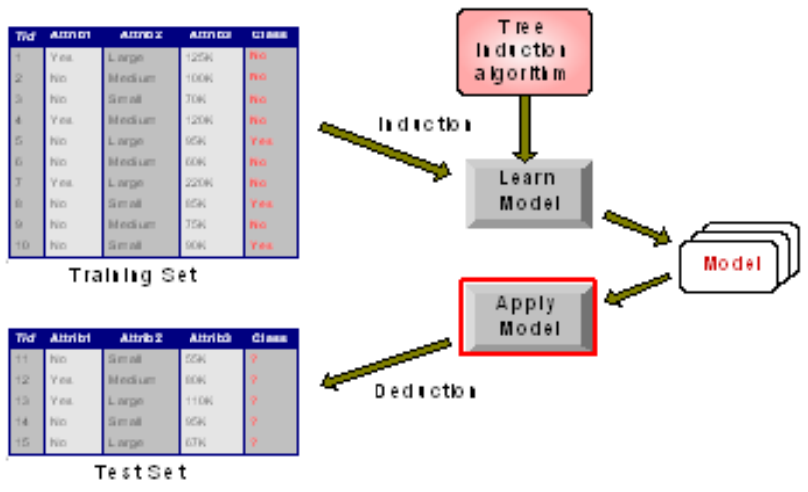

Fig 1: KNN based BABC test and training technique wellknown classification

This will provide us with a clearly defined object which can be labeled removed. After BABC (Tree indicator) algorithms testing and training processes independently.

\section{K-NEAREST-NEIGHBOR CLASSIFIER}

k-Nearest Neighbor (KNN) category is one of the simplest category strategies, and it ought to the primary selections for a class examine whilst there's no previous knowledge about the distribution of the records. KNN type turned into advanced from the discriminate analysis[20], which is used to perform whilst reliable parametric estimates the chance densities which might be unknown or tough to determine. It makes use of Euclidean distance as the gap dimension. For comparing the accuracy of the selected subset, the KNN classifier acts because the health evaluator.

As a similarity-based totally method, the k-nearest neighbor classifier (ok-NN) is applying to our multidimensional category. First, the classifier calculates ok maximum comparable files (i.e., okay nearest buddies) of the check attributes is classed. The similarity of these attributes to a category is computed with the aid of summing up the similarities of attributes a few of the okay attributes, whose lessons are equivalent to such magnificence. The take a look at the record is assigned the magnificence that has the very best similarity to the attributes. The parameters involved are the definition of similarity and the range $\mathrm{k}$.

While the standard similarity is defined as TFIDF (term frequency-inverse document frequency), a variant Certainty of a rule can be measured with a threshold of confidence. This parameter lets to measure how often an event's order sets that match the left side of the implication of the association rule also matches for the right side that performed better in our preliminary experiments, is applied in this work. The parameter $\mathrm{k}$ is dictated by the tests as appeared in the following segment.

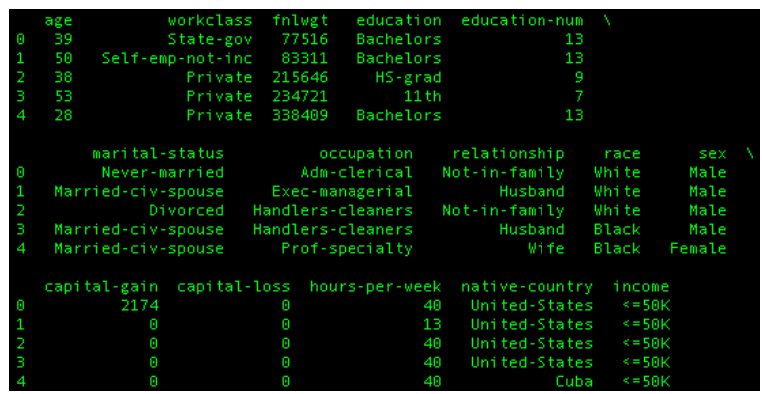

Fig 1(A) demonstrates the proposed philosophy structure. 
To start with the records is reprocessed into part token (Test and Preparing). After token ionization, stemming process is connected to acquire the root words. At that point, Stopwords, for example, article's relational words are expelled from the dataset. Highlights are removed utilizing Sententious. At that point the choose highlights are changed into Extraction by utilizing TFIDF.

We start by KNN classification using the training set of all BABC algorithms with outliers and giving the individual elements some sort of order. Let $\mathrm{X}$ be the training set and $\mathrm{P}$ be what we call "the set of prototypes". We then scan all elements of $\mathrm{X}$ and move individual elements to $\mathrm{P}$ if their nearest prototype (their nearest element from $\mathrm{P}$ ) has a different class label.

1. Starting with $\mathrm{P}=\{\mathrm{x} 1\}$, we then repeat this algorithm until no more prototypes are added to $P$.

2. We then describe a point as "absorbed" if it is not a prototype, and call a point an "outlier" if none of its $\mathrm{k}$ nearest points share the same class.

3. Outliers are points whose $\mathrm{k}$ nearest points are not the same class.

4. $\mathrm{X}=\{\mathrm{x} 1, \mathrm{x} 2, \ldots, \mathrm{xn}\}$ (without outliers).

5. $\mathrm{P}=\{\mathrm{x} 1\}$ to $\mathrm{c} 1, \mathrm{c} 2, \mathrm{c} 3$;

6. We scan all elements of $\mathrm{X}$ and move individual elements to $\mathrm{P}$ if their nearest prototype (their nearest element from $\mathrm{P}$ ) has a different class label.

7. Repeat until no more new prototypes are found.

8. Absorbed points are the points which are not prototypes.

9. Prototypes are denoted by squares.

10. Outliers are denoted with crosses.

11. Absorbed points are denoted by empty circles.

Absorbed points and outliers are $\mathrm{KNN}$ used for $\mathrm{BABC}$ algorithms classification, Training maps that are created multi dimensionreduce type of testing. The k-closest neighbor calculation groups objects in light of a larger part vote of the $\mathrm{k}$ closest preparing and testing.

We assign the class label which is the most frequent amongst the $\mathrm{k}$ training examples which are nearest or most similar to our previously unseen instance.

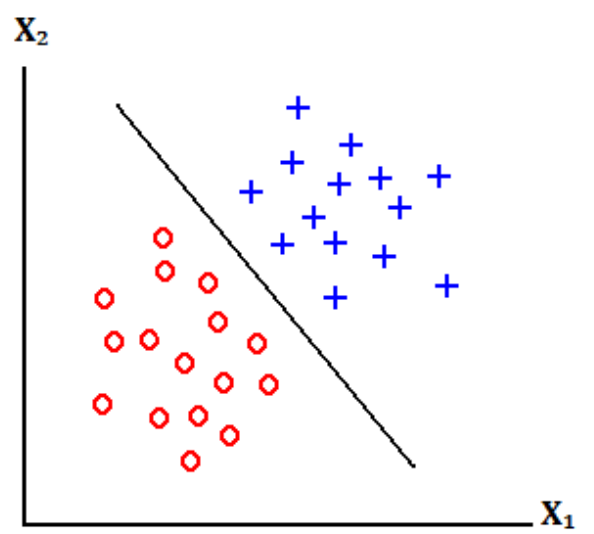

Fig:2 Data sets feature Extraction

- Feature Extraction of $\mathrm{x}_{1}$ and $\mathrm{x}_{2}$ and two classes represented by $x$ and $o$.

- Each x\&o speak to one example in include space characterized by its estimations of $\mathrm{x} 1$ and $\mathrm{x} 2$.

Two dimensional Gaussian data with two classes (substantial within class covariance).

\section{Estimates subspace for global dimension reduction.}

From the local neighborhood N(i) of xi, the local class centroids are contained in a subspace useful for classification. Each preparation point xi, the betweencentroids aggregate of square lattice $\mathrm{Bi}$ is processed, and afterward these networks are finding the middle value of over all preparation focuses:

1. The eigenvectors e1, e2,...ep of the matrix span the optimal subspaces for global subspace reduction preprocessing dataset.

2. A Dataset may need to be preprocessed to ensure more reliable data mining results.

3. Conversion of non-numeric data to numeric data

4. Calibration of numeric data to reduce the effects of disparate ranges

5. Particularly when using the Euclidean distance metric.

\section{Applied BABC Multidimensional}

It is all the binary values zero, one value objects in the Classification are labeled; we can analysis each object as a binary Classification. The marked protest has an estimation of ' 1 ' and everything else is ' 0 '. The naming procedure goes as takes after:

1. Define the coveted network.

2. Scan the Order and mark associated objects with a similar image.

3. After we have named the items, we have an Order loaded with protest numbers.

4. This Order is utilized to extricate the highlights of intrigue.

5. Among the double protest highlights incorporate region, the focal point of the zone, pivot of slightest second minute, edge, Euler number, projections, slimness apportion and angle proportion.

6. In request to extricate those highlights for singular question, we have to make isolate twofold Grouping for each of them.

7. We can accomplish this by doling out 1 to pixels with the predetermined name and 0 somewhere else.

8. If after the naming procedure, we wind up with 2 distinctive labels(zero, one), at that point we have to make 2 isolate double Qualities for each question.

\section{BABC Algorithm}

1. Initialize the confidence $\sum=\mathrm{I}$, the identity supports

2. KNN regression: $Y=\alpha+\beta X$

3. Testing: Two parameters , $\alpha$ and $\beta$ specify the line and are to be estimated by using the data at hand.

4. Training: KNN using the least squares criterion to the known values of $\mathrm{Y} 1, \mathrm{Y} 2$, $\ldots, \mathrm{X} 1, \mathrm{X} 2$.

5. KNN. regression: $\mathrm{Y}=\mathrm{b} 0+\mathrm{b} 1 \mathrm{X} 1+\mathrm{b} 2$ 
X2.

6. Many nonlinear functions can be transformed into the above.

7. $\quad$ KNN. $($ Dataset $)=$ Prune $($ Tree_Generation $($ Dataset $))$

8. KNN.Tree_Generation (Dataset) $=$ IF termination_condition (Dataset)

9. THEN leaf ( majority_class (Dataset) )

10. Training data is of the form $\left(\mathbf{X}_{1}, \mathrm{y}_{1}\right),\left(\mathbf{X}_{2}, \mathrm{y}_{2}\right), \ldots$, $\left(\mathbf{X}_{|\mathbf{D}|}, \mathbf{y}_{|\mathrm{D}|}\right)$

11. Testing.2-D data, we may have: $\mathrm{y}=\mathrm{w}_{0}+\mathrm{w}_{1} \mathrm{x}_{1}+\mathrm{w}_{2}$ $\mathrm{x}_{2}$

12. ELSE IF.

13. LET(Testing. Training $=100 \%$ )

14. Best test $=$ FE_function (Dataset)

15. IN Node

16. Training data : error_training $(\mathrm{h})$

17. Entire distribution D of data : error_D (h)

18. BABC (error_training $(\mathrm{h})<$ error_training (h'))

19. error_D $(h)>$ error (h')

20. FOR EACH value $\mathrm{v}$ OF Best_test

21. Let subtree_v $=$ Tree_Generation ( $\{$ e e example $\mid$ e.Best_test $=\mathrm{v})$

22. IN Node (Best_test, subtree_v )

23. Comparison $\mathrm{NB}=$ Best.Training+Test;

24. Result of BABC Precision;

The Proposed highlight extraction technique utilizing Paired Simulated Honey bee Settlement (BABC)

Double Simulated Honey bee State (BABC) comprises of three honey bees to be specific utilized honey bees, passerby honey bees and scout honey bees. The honey bees move around the pursuit space to locate the ideal arrangements. Here are features are represented as the details sources and nectar amount represent the human details value (T.ID, Refund, married status,chat) of each confidence source.

In the component extraction issue, the competitor arrangements are spoken to by parallel piece string N, where $\mathrm{N}$ speaks to the aggregate number of highlights. In the event that the incentive at the relating position is 1 then the element is chosen as a component of the subset to be assessed or something bad might happen if the esteem is 0 then the element isn't Extraction as a major aspect of the subset. The component Extraction subset of every nourishment source is given to the classifier to ascertain the wellness esteem (nectar sum).

\section{Steps of BABC Algorithm:}

1. Partition attributes into sub-patterns.

2. Compute the expected contribution of each sub-pattern. Generate the Mean and Median faces for each person, and use these "virtual attributes" as the probe set in training.

3. Use the raw attributes Classification sub-patterns as the gallery set in for training, and compute the $\mathrm{KNN}$ projection matrix on these galleries set BABC.

4. For each sample in the probe set, compute its similarity to the samples in the corresponding gallery set

Where $\mathrm{X}_{\text {mini }}, \mathrm{X}_{\text {minj }}$ are the lower and upper bound of dimension j. Evaluate the Fitness value using the accuracy:

$$
X_{i j}=X_{\text {minj }}+\operatorname{rand}\left(X_{\text {mimi }}-X_{\text {minj }}\right)(1)
$$

$$
\begin{aligned}
\text { Accuracy }=\frac{\# \text { Support }}{\text { Addupto } \# \text { certainty }} \\
\quad=\frac{A_{11}+A_{00}}{A_{11}+A_{10}+A_{01}+A_{00}}
\end{aligned}
$$

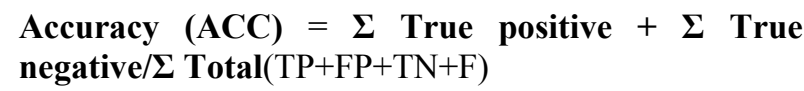

Where $\mathrm{TP}=$ True Positive $\mathrm{TN}=$ True Negative, $\mathrm{FP}=$ False Positive, $\mathrm{FN}=$ False Negative.

5. BABC Classification: When an unknown Classification Attribute comes in

a. Partition it into sub-patterns.

b. Classify the unknown sample's identity in each subpattern.

c. Incorporate the expected contribution and the classification result of all sub-patterns to generate the final classification result

$$
\hat{k}=\frac{N \sum_{i=1}^{r} x_{i i}-\sum_{i=1}^{r}\left(x_{i+} \cdot x_{+i}\right)}{N^{2}-\sum_{i=1}^{r}\left(x_{i+} \cdot x_{+i}\right)}
$$

6. Dataset

a. KNN Classification database: 1400 Attributes of 500 males and 500 females, each person has 400 Attributes.

b. BABC Classification database: 1650 Attributes of 150 adults, 100 Attributes per person.

c. KNN+BABC Classification database: 1400 Attributes of 400 adults, 100 Attributes per person .

7. If a sample from a sub-pattern's probe set is correctly classified, the contribution of this sub-pattern is added by 1

8. $\mathrm{N}=\mathrm{a}+\mathrm{b}+\mathrm{c}+\mathrm{d}$

9. Accuracy $=(a+d) / N$

Cost $=p(a+d)+q(b+c)$

$=\mathrm{p}(\mathrm{a}+\mathrm{d})+\mathrm{q}(\mathrm{N}-\mathrm{a}-\mathrm{d})$

$=\mathrm{q} N-(\mathrm{q}-\mathrm{p})(\mathrm{a}+\mathrm{d})$

$=\mathrm{N}[\mathrm{q}-(\mathrm{q}-\mathrm{p}) \times$ Accuracy $]$

10. The area $A_{i}$ is measured in pixels and indicates the relative size of the object. $A_{i}=\sum_{r=0}^{\text {height }-1} \sum_{c=0}^{\text {width-1 }} I_{i}(r, c)$

11. These correspond to the row and column coordinate of the center of the ith object. $\bar{r}_{i}=\frac{1}{A_{i}} \sum_{r=0}^{\text {height }-1} \sum_{c=0}^{\text {width }-1} r I_{i}(r, c)$

$\bar{C}_{i}=\frac{1}{A_{i}} \sum_{r=0}^{\text {height }-1} \sum_{c=0}^{\text {width }-1} c I_{i}(r, c)$

12. The Axis of Least Second Moments are expressed as $\theta$ - the angle of the axis relatives to the vertical axis 
$\theta_{i}=\frac{1}{2} \tan ^{-1}\left(\frac{2 \sum_{r=0}^{\text {height }} \sum_{c=0}^{\text {width-1 }}(r-\bar{r})(c-\bar{c}) I_{i}(r, c)}{\sum_{r=0}^{\text {height } 1 \text { widtth1 }} \sum_{c=0}(r-\bar{r})^{2} I_{i}(r, c)-\sum_{r=0}^{\text {height } \sum_{c=0}^{\text {width } 1}(c-\bar{c})^{2} I_{i}(r, c)}}\right)$ This

assumes that the origin is as the center of the area.

a. This includesgiving data about the protest's introduction.

13. This hub compares to the line about which it takes minimal measure of vitality to turn a protest.

14. The border is characterized as the aggregate pixels that constitutes the edge of the protest.

15. Perimeter can help us to find the protest in space and give data about the state of the question.

16. Perimeters can be found by tallying the quantity of ' 1 ' pixels that have ' 0 ' pixels as neighbors.

17. Perimeter can likewise be found by applying an edge finder to the protest, trailed by checking the '1' pixels.

18. The two strategies above, just give a gauge of the genuine border.

19. An enhanced gauge can be found by increasing the outcomes from both of the two techniques by $\pi / 4$.

20. The slenderness proportion, $\mathrm{T}$, can be ascertained from the edge and region.

21. The condition for slenderness proportion is characterized as takes after:

22. The slimness proportion is utilized as a measure of roundness. It has a most extreme estimation of 1 , which relates to a circle.

23. As the protest ends up plainly more slender and more slender, the edge ends up noticeably bigger in respect to the zone and the proportion diminishes and emerge exactness.

\section{EXPERIMENTAL RESULTS}

The evaluation of the proposed method was carried out using the movie review dataset is collected UCI REALDISP(PSO,ABC) Activity Recognition Data set[6]. It contains 2000 reviews consists of 1000 positive and 1000 negative reviews[2]. The dataset is divided into training and testing data set. From this review dataset, 82\% (1640 instances) of the reviews are taken from the training data set stand the other $18 \%$ (360instances) of reviews are taken fromthe testing dataset with the significant features.

Table I BABC Dataset Parameters

\begin{tabular}{|l|l|l|}
\hline Total Data Set & Testing & Training \\
\hline 2000 & $82 \%$ & $18 \%$ \\
\hline 2000 & 1640 & 360 \\
\hline & & \\
\hline Test and Training & Class=Yes & Class=No \\
\hline Class=Yes & 1600 & 40 \\
\hline Class=No & 40 & 320 \\
\hline
\end{tabular}

\section{Estimate Metrics:}

The estimate of the proposition BABC_KNN was carried out using the following metrics.

$$
\text { Accuracy }=\frac{a+d}{a+b+c+d}=\frac{T P+T N}{T P+T N+F P+F N}
$$

Where, $\mathrm{TN}=$ True Positive $\mathrm{FP}=$ False Positive $\mathrm{FN}=$

False Negative. $\mathrm{TP}=$ True Positive.

\section{BABC_KNN consideration settings:}

With a specific end goal to diminish the computational time, the settlement measure is set to 40 and the most extreme number of emphases is set to be at 100 . In the wake of introducing the parameters, Highlights subsets are taken as the contribution for the classifier. With a specific end goal to assess the wellness (Precision) of both the representative stage and passerby stage, we utilize two classifiers to be specific $\mathrm{KNN}$ and BABC classifier to assess the chose subsets of highlights for every datum set[10]. For each situation, a 10 cross folds approval is utilized.

The Table I, shows the parameters used for the algorithm and the Table II, shows the selected features in different runs and the accuracy for the PSO(Best Practice Spotlight Organizations) and the modified PSO uses KNN and BABC (NB) Classifier.

Parameter Setting:

Fig 2 BABC Parameters

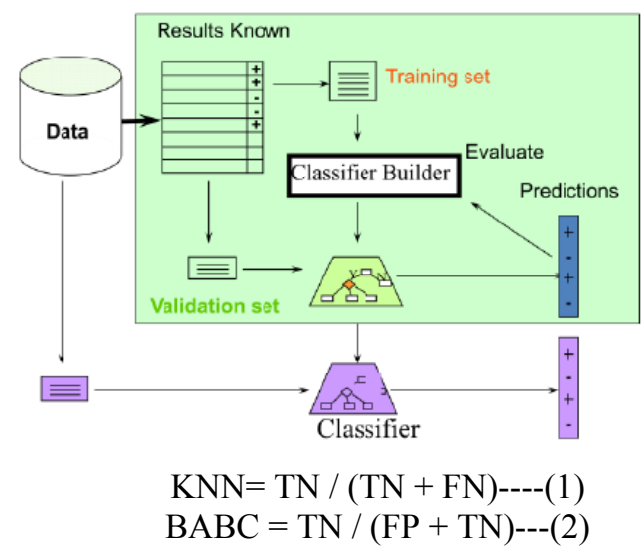

Table II Classification accuracy

\begin{tabular}{|c|c|c|c|}
\hline Classifier & PSOFeatures & ABCFeatures & $\begin{array}{c}\text { Proposed } \\
\text { BABCFeatures }\end{array}$ \\
\hline KNN & 85.5 & 88.25 & 98.22 \\
\hline NB & 82.5 & 85.46 & 88.84 \\
\hline
\end{tabular}

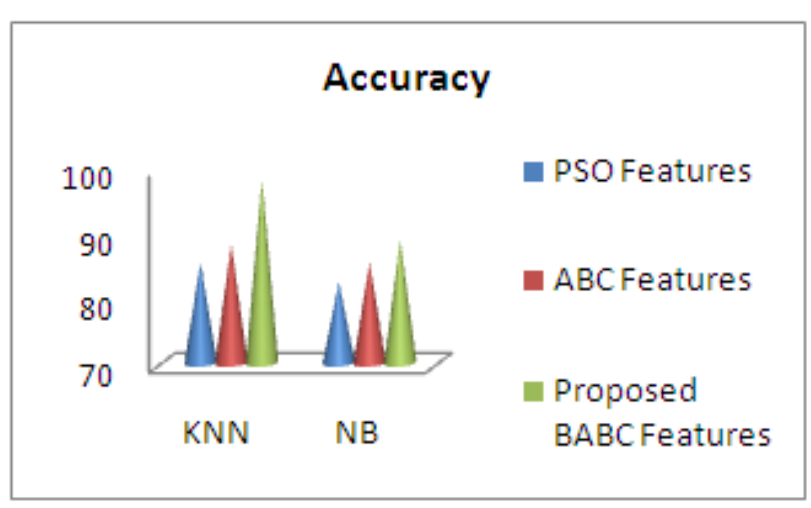

Figure 3 Accuracy 
From Table II and fig. 3 , it is watched that Exactness is enhanced for proposed BABC for include extraction when contrasted with PSO highlights and ABC highlights. On a normal, Precision increments for Proposed BABC by $12.72 \%$ when contrasted with PSO, by $9.97 \%$ when contrasted with ABC.

Tables III and Table IV demonstrate the normal accuracy, review acquired for different element extraction. The outcomes are appearing Fig 4 and Fig 5.

Table III Precision

\begin{tabular}{|l|c|c|c|}
\hline Classifier & $\begin{array}{c}\text { PSO } \\
\text { Features }\end{array}$ & $\begin{array}{c}\text { ABC } \\
\text { Features }\end{array}$ & $\begin{array}{c}\text { Proposed BABC } \\
\text { Features }\end{array}$ \\
\hline KNN & 87.5 & 89.65 & 96.00 \\
\hline NB & 87.5 & 86.66 & 89.92 \\
\hline
\end{tabular}

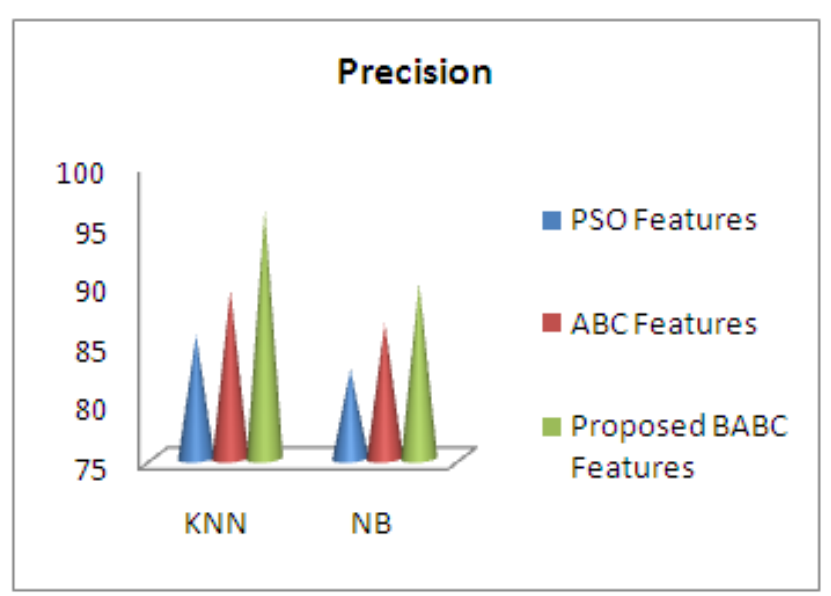

Figure 4 Precision

From Table III and Fig 4, it is watched that Accuracy is enhanced for Proposed BABC for highlight extraction when contrasted with PSO(random measurement selection)features and $\mathrm{ABC}$ highlights. On a normal, Exactness increments for Proposed BABC by $10.5 \%$ when contrasted with PSO, by $6.75 \%$ when contrasted with ABC.

Table IV Recall

\begin{tabular}{|l|l|l|l|}
\hline Classifier & $\begin{array}{l}\text { PSO } \\
\text { Features }\end{array}$ & $\begin{array}{l}\text { ABC } \\
\text { Features }\end{array}$ & $\begin{array}{l}\text { Proposed BABC } \\
\text { Features }\end{array}$ \\
\hline KNN & 81.5 & 89.25 & 96.48 \\
\hline NB & 77.58 & 86.46 & 89.88 \\
\hline
\end{tabular}

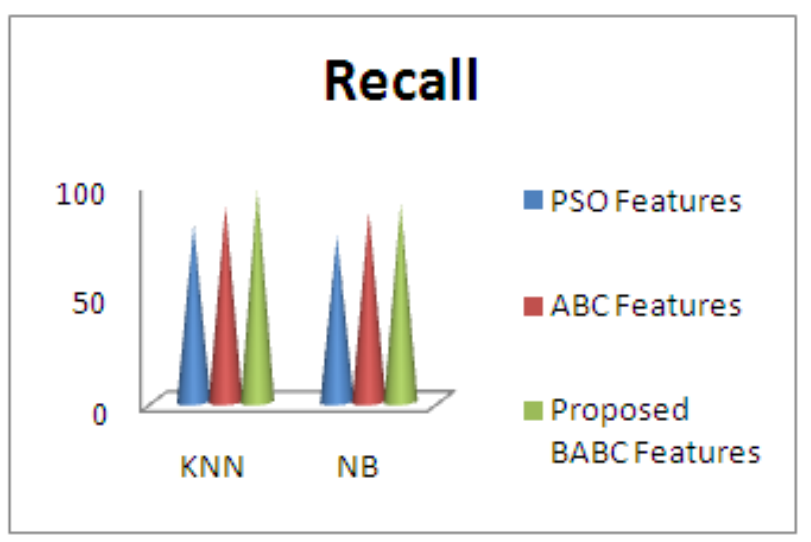

Figure 5 Recall
From Table IV and Fig 5, it is watched that theRecall is enhanced for Proposed BABC for highlight extraction when contrasted with PSO highlights and ABC highlights. On a normal, Review increments for Proposed BABC by $15.98 \%$ when contrasted with PSO, by $7.23 \%$ when contrasted with $\mathrm{ABC}$

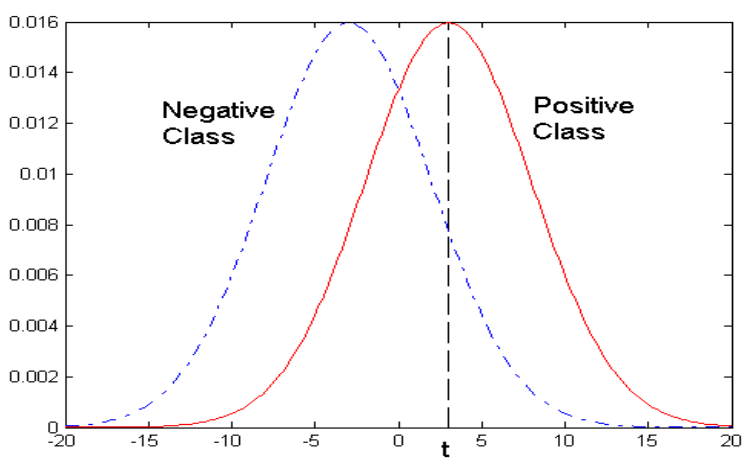

Fig 6: BABC based NC vs PC

Multidimensional data set containing 2 classes (positive and negative) any points located at $\mathrm{x}>\mathrm{t}$ is classified positive.

\section{$\mathrm{TP}=0.5, \mathrm{FN}=0.5, \mathrm{FP}=0.12, \mathrm{FN}=0.88$}

\section{Measurements}

(TP,FP):

$(0,0)$ : Declare everything to be negative class

$(1,1)$ : Declare everything to be positive class

$(1,0)$ : Ideal

$(0,1)$ :Starts Class.

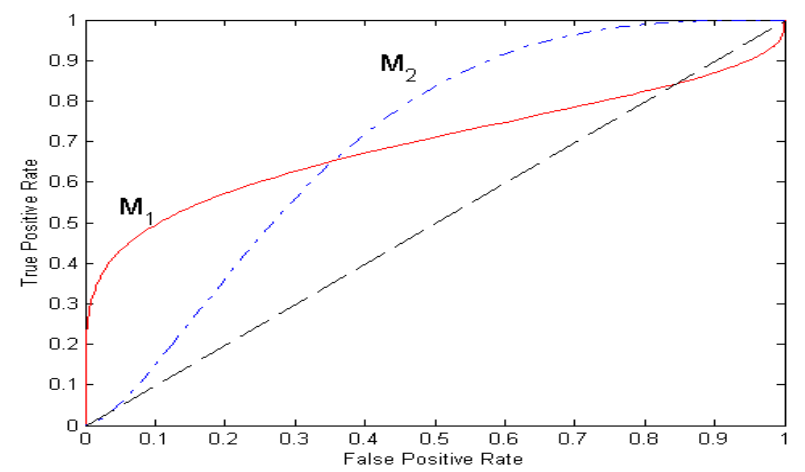

Fig.7 ROC TPR and FPR

No model consistently outperformsthe other

o $\quad \mathbf{M}_{\mathbf{1}}$ is better for small FPR

o $\quad \mathbf{M}_{2}$ is better for large FPR

- $\quad$ Area Under the ROC curve (AUC)

o Ideal: Area $=1$

o Random guesses:

- Binary classification

$$
\text { - } \quad \text { Area }=0.5
$$

o (Instances, Class labels): $\left(\mathrm{x}_{1}, \mathrm{y}_{1}\right),\left(\mathrm{x}_{2}, \mathrm{y}_{2}\right)$, $\ldots,\left(\mathrm{x}_{\mathrm{n}}, \mathrm{y}_{\mathrm{n}}\right)$

o $\mathrm{y}_{\mathrm{i}}\{1,-1\}$ - valued

Classifier: provides class prediction $\hat{Y}$ for an instance 
Table V: Comparison Table

\begin{tabular}{|c|c|c|c|c|}
\hline Classifier & $\begin{array}{c}\text { Multidi } \\
\text { mensio } \\
\text { nal } \\
\text { Dataset }\end{array}$ & $\begin{array}{c}\text { Number } \\
\text { of } \\
\text { Test } \\
\text { Attribut } \\
\text { es }\end{array}$ & $\begin{array}{c}\text { Correctl } \\
\mathbf{y} \\
\text { Classifie } \\
\mathbf{d} \\
\text { Attribut } \\
\text { es }\end{array}$ & $\begin{array}{c}\text { Classific } \\
\text { ation } \\
\text { Rate (\%) }\end{array}$ \\
\hline \multirow{2}{*}{$\begin{array}{c}\text { KNN+BA } \\
\text { BC }\end{array}$} & $\begin{array}{c}\text { ABC } \\
\text { Dataset }\end{array}$ & 1200 & 1160 & 96.66 \\
\cline { 2 - 5 } & $\begin{array}{c}\text { PSO } \\
\text { Dataset }\end{array}$ & 1200 & 1150 & 95.83 \\
\hline \multirow{2}{*}{ KNN } & $\begin{array}{c}\text { ABC } \\
\text { Dataset }\end{array}$ & 1200 & 1020 & 85 \\
\cline { 2 - 5 } & $\begin{array}{c}\text { PSO } \\
\text { Dataset }\end{array}$ & 1200 & 980 & 81.66 \\
\hline \multirow{2}{*}{ NP } & $\begin{array}{c}\text { PSO } \\
\text { Data set }\end{array}$ & 1200 & 990 & 82.5 \\
\cline { 2 - 5 } & $\begin{array}{c}\text { ABC } \\
\text { Dataset }\end{array}$ & 1200 & 940 & 78.33 \\
\hline
\end{tabular}

The performance of the BABC is better than KNN and NP. Table V explains the Comparison of algorithms - BABC, $\mathrm{KNN}$ and NP. The performance analysis of the algorithms using Multidimensional to show the algorithm BABC is better than KNN and NP in extracting the details according to the user's need with better accuracy.

\section{CONCLUSION}

In this paper, BABC-KNN based component extraction has been utilized to characterize the surveys into positive and negative audits. The proposed BABC based element extraction gives ideal component subset. Assess the precision of the chose subset of highlights, the KNN calculations is utilized as the classifier. The test comes about demonstrate that the proposed BABC with $\mathrm{k}-\mathrm{NN}$ chooses the negligible number of highlights with the most astounding arrangement precision and lessens the computational many-sided quality contrasted and other list of capabilities.

\section{REFERENCES}

1. Lin $\mathrm{Y}$, Zhu T, Wang X, Zhang J, \& Zhou A, "Towards online anti-opinion spam: Spotting fake reviews from the review sequence",In 2014 IEEE/ACM international conference on Advances in social network analysis and Mining (ASONAM) pp 261-264, IEEE,2014.

2. AshwiniHambarde1, S.P. Kosbatwar,"Efficient Feature by Using Global Redundancy Minimization and Constraint Score", International Research Journal of Engineering and Technology (IRJET), Volume: 02 Issue: 09 | Dec-2015

3. Aksu Y, Miller D J and Kesidi G, "Margin- maximizing feature elimination methods for Linear and nonlinear kernelbased discriminant functions“, IEEE Trans. Neural Networks, vol. 21, pp.701-717, 2010.

4. Gutlein M, Frank E and Karwath A, "Large- scale attribute extraction using wrappers“, In IEEE Symposium Computational Intelligence and Data mining, pp. 332$339,2010$.

5. Alper U, Alper M and Ratna B C, "PSO:A maximum relevance minimum redundancy feature extraction method based on swarm intelligence for support vector machine classification", Information Science, vol. 181, pp. 4625-4641,2011.

6. Vieira S, Luis M, MendoncaF, Farinha LF, and Sousa M C, "Modified Binary PSO for feature extraction using SVM applied to mortality prediction of septic patients", Applied soft computing vol. 13, pp. 3494-3504,2013.

7. Zhang, X., \& Zhang, X, " A binary artificial bee colony algorithm for Constructing spanning trees in vehicular Ad hoc Networks“, Ad Hoc Networks,58, 198-204,2017.

8. Zhang, X, Zhang, X, \&Gu C. (2017), "A micro-artificial bee colony based multicast routing in vehicular Ad hoc networks and Ad Hoc Networks", 58, 213-221,2017.

9. Zabidi, Azlee,MdTahir, Nooritawati,Mohd, Ihsan and Rizman, Zaire, " The performance of the binary artificial bee colony (BABC) in structure selection of polynomial NARX and NARMAX models",International Journal on Advanced Science, Engineering and Information Technology. 7. 373-379. 10.18517/ijaseit.7.2.1387.

10. ThanananPrasartvit, Anan Banharnsakun, BoonsermKaewkamnerdpong and TiraneeAchalakul,"Reducing Bio- informatics data dimension with ABC-K-NN", Neurocomputing, vol. 116, pp. 367-381, 2013.

11. Sung-Soo Kim, Ji-Hwan Byeon ,Ajith Abraham," Optimal job scheduling in grid computing using efficient binary artificial bee colony optimization ". Published online: 20 November 2012, Springer-Verlag Berlin Heidelberg 2012

12. Chatterjee, A., Tudu, B. \& Paul, "Binary grayscale halftone pattern generation using binary artificial bee colony (BABC)",K.C. (2013)7:1195.https://doi.org/10.1007/s11760-012-0388-z

13. Pampara G, Engelbrecht A P, “ Binary artificial bee colony optimization“, In IEEE Symposium on Swarm Intelligence, IEEE, Perth, pp. 1-8(2011).

14. Kashan M H, Nahavandi N, and Kashan AH, " A new artificial bee colony algorithm for binary optimization",Applied Soft Computing, 12(1), pp. 342-352(2012).

15. OzturkandC,Karaboga D, "HybridArtificialbeecolony algorithm for neural network training", In Evolutionary Computation (CEC), 2011 IEEE Congress on, pages 84-88. IEEE,2011.

16. Fei Kang, JunjieLi, HaojinLi, Zhenyue Ma and Qing Xu, "An Improved Artificial Bee Colony Algorithm", IEEE International

WorkshoponIntelligentSystemsandApplications,2010,pp14,2010 .

17. Xiao, Y., $\mathrm{Yu}$ W,"Bee Colony algorithm for image edge detection", Appl. Res. Comput. 27(7), 2748-2750 (2010).

18. SP.Rajamohana,Dr.K.Umamaheswari, "Feature Selection classification",International Research Journal of Engineering and Technology,Volume: 03 Issue: 12, 2017.

19. Liu, T., Zhang, L., Zou, K., et al,"Multiuser detection based on differential evolution binary Artificial Bee Colony algorithm.“,Adv. New Renew. Energy 18(1), 5-10 (2013)

20. Rajamohana SP \&Umamaheswari K, "Sentiment Classification based on LDA using SMO Classifier", International Journal of Applied Engineering Research, ISSN 0973-4562 Vol. 10, No.55 ,pp. 1045-1049,2015. 